\title{
Preoperative assessment for scheduling surgery during the coronavirus disease pandemic
}

\author{
Koichi Suehiro ${ }^{1}[\mathbb{D}$
}

Received: 13 October 2020 / Accepted: 8 January 2021 / Published online: 27 January 2021

(c) Japanese Society of Anesthesiologists 2021

\begin{abstract}
On Mar 11, 2020, the World Health Organization declared coronavirus disease (COVID-19), caused by severe acute respiratory syndrome coronavirus-2 (SARS-CoV-2), a pandemic. Because COVID-19 has a pre-symptomatic period of up to 2 weeks, SARS-CoV-2 infection has continued to spread. Some individuals with SARS-CoV-2 infection have a severe clinical course, while most individuals have mild or moderate symptoms. Because SARS-CoV-2 is transmitted via droplets and secretions, anesthesiologists have higher risks of infection, especially during airway management. Therefore, general anesthesia requiring airway management can be a challenging procedure for anesthesiologists. During the pandemic, many elective surgeries have been postponed or cancelled in most affected countries. Recently, the number of elective surgeries is gradually recovering from the effect of the COVID-19 pandemic, and hence, safe clinical practice and protocols to prevent SARS-CoV-2 transmission to medical staff should be established. This mini-review focuses on the preoperative assessment and decision with regard to scheduling surgery in elective and emergency cases during the COVID-19 pandemic. A standardized questionnaire and algorithm regarding COVID-19 should be used to assess surgical patients preoperatively as it increases the reproducibility and accuracy of the decision whether to proceed with surgery.
\end{abstract}

Keywords COVID-19 $\cdot$ SARS-CoV-2 $\cdot$ Surgery $\cdot$ Anesthesia

\section{Introduction}

The World Health Organization declared coronavirus disease (COVID-19), which is caused by severe acute respiratory syndrome coronavirus 2 (SARS-CoV-2), a global pandemic on Mar 11, 2020. As of Sep 2, 2020, over 69,000 people have been infected with SARS-CoV-2 in Japan, with a mortality rate of approximately $1.9 \%$ (Ministry of Health, Labour and Welfare. About Coronavirus Disease 2019 (COVID-2019). https://www.mhlw.go.jp/stf/seisakunitsuite/bunya/newpa ge_00032.html; accessed: Sep 3, 2020). COVID-19 has continued to spread, because it has a pre-symptomatic period of up to 2 weeks during which SARS-CoV-2 can be transmitted to other individuals [1]. Most SARS-CoV-2-infected

Koichi Suehiro

suehirokoichi@yahoo.co.jp

1 Department of Anesthesiology, Osaka City University Graduate School of Medicine, 1-5-7 Asahimachi, Abenoku, Osaka, Osaka 545-8586, Japan individuals have mild or moderate symptoms; however, some individuals have a severe clinical course [2]. Because SARS-CoV-2 transmission increases with exposure to droplets and secretions, anesthesiologists have higher risks of SARS-CoV-2 transmission during procedures, including mask ventilation, tracheal intubation, extubation, tracheostomy, and bronchoscopy [3, 4]. Therefore, general anesthesia requiring airway management can be challenging for anesthesiologists [5]. Since the start of the pandemic, many elective surgeries have been postponed or cancelled in most affected countries. However, recently, the number of elective surgeries is gradually recovering from the effect of the COVID-19 pandemic; hence, safe clinical practice and protocols to prevent transmission from patients to medical staff need to be established. This mini-review focuses on the preoperative assessment and decision with regard to scheduling surgery during the COVID-19 pandemic. 


\section{Testing for COVID-19}

Testing for COVID-19 is essential to protect patients and medical staffs from infection risk, especially during the perioperative period. The infection of SARS-CoV-2 in surgical patients can lead to increased morbidity and mortality. A previous study [6] investigated 1128 surgical patients (elective surgery: $24.8 \%$, emergency surgery: $74.0 \%$ ) who had SARS-CoV-2 infection confirmed within 7 days before or 30 days after surgery. In this cohort, the mortality rate was $23.8 \%$, and pulmonary complications including acute respiratory distress syndrome, pneumonia and unexpected postoperative ventilation occurred in $51.2 \%$.

At the moment, a polymerase chain reaction (PCR) test has been considered as a gold-standard technique for diagnosis of COVID-19. Generally, diagnostic tests can cause inaccurate results in the following two ways. False positive results can lead to unnecessary protecting and contact tracing. False negative results which erroneously label COVID-19 patients uninfected can be more consequential, because the patients may not be isolated if they are asymptomatic [7]. A recent systematic review indicated the estimate of the false negative rate of the PCR test was 0.13 (95\% CI: 0.09-0.19) [8]. Another meta-analysis showed a pooled sensitivity of the PCR test as 89\% [95\% confidence interval (CI): 81-94\%] [9]. The sensitivity of the PCR test was negatively correlated with the proportion of elderly patients $(p=0.01)$ [9]. The positive predictive value ranged from 47.3 to $96.4 \%$, while the negative predictive value ranged from 96.8 to $99.9 \%$ [9]. However, the physicians have to realize that the positive and negative prediction values depend on the infection probability of the patient as well as the sensitivity and specificity of the test [10].

The American Society of Anesthesiologist and the Anesthesia Patient Safety Foundation recommend that patients undergoing elective surgery should be tested for COVID-19 shortly before the scheduled procedure, especially in areas with high epidemic status [11]. If the capabilities of COVID-19 testing are limited and the local epidemic status is low, the screening for symptoms and exposure to COVID-19 contacts can be considered [12].

\section{Pre-anesthetic consultation}

The joint task force of the Chinese Society of Anesthesiology and the Chinese Association of Anesthesiologists published a recommendation regarding precautions during pre-anesthetic consultation [13]. The task force recommended that medical staff should wear personal protective equipment, including surgical masks, eye protection shields, and medical gloves, during the pre-anesthetic consultation [13]. Patients are required to enter the anesthetic consulting room one at a time to minimize the frequency of contact with medical staff and other patients. Patient's body temperatures should be measured before entering the consulting room, and only those with a normal body temperature should be allowed to enter. The task force recommended that patients with a high body temperature $\left(>37.3{ }^{\circ} \mathrm{C}\right.$ ) should be transferred to the medical clinic for fever disorders, and medical staff should report the patient to the infection control center [13]. Anesthesiologists should change their gloves and sanitize their hands (2-3\% hydrogen peroxide) after contact with each patient [13]. If patients are suspected to have COVID-19, medical staff should report the patient to the infection control center immediately [13].

\section{Standardized questionnaire}

The French Society of Anaesthesia and Intensive Care Medicine introduced guidelines regarding the preoperative strategy for COVID-19 in surgical patients [14]. The guidelines recommended using a standardized questionnaire regarding COVID-19 to preoperatively assess surgical patients, because it increases the reproducibility and accuracy of medical examinations [14]. The data obtained from a large number of patients must be easily traceable and quantifiable. The standardized questionnaire should be constructed as easily understood by a variety of patients. An example of a standardized questionnaire has been provided in the guidelines [14], which includes questions regarding five major symptoms (fever, dry cough, difficult breathing, loss of smell, and loss of taste) and ten minor symptoms (sore throat, rhinorrhea, chest pain, pain in the muscle, severe fatigue, confusion, headache, diarrhea, nausea and vomiting, and a rash or cracked skin on the fingers or hands). Fever is one of the most common symptoms of symptomatic COVID-19 and is present in many of cases [14-16]. Thus, the body temperature needs to be measured during the pre-anesthetic consultation. Data on the intake of antipyretic drugs, including non-steroidal anti-inflammatory drugs, should be systematically collected, as these drugs can normalize the temperature of febrile patients. Fever is an important warning sign and thus, the recent guidelines recommended that the body temperature should be measured repeatedly until the day of the surgery [14].

\section{Preoperative strategy for coronavirus disease in patients undergoing scheduled surgery}

The guidelines by the French Society of Anaesthesia and Intensive Care Medicine introduced two algorithms 
Fig. 1 Preoperative assessment of patients undergoing scheduled surgery. Adapted from Velly et al. 2020 [13]. Abbreviations: COVID-19 coronavirus disease, $C T$ computed tomography, $P C R$ polymerase chain reaction, SARS-CoV-2 severe acute respiratory syndrome coronavirus 2

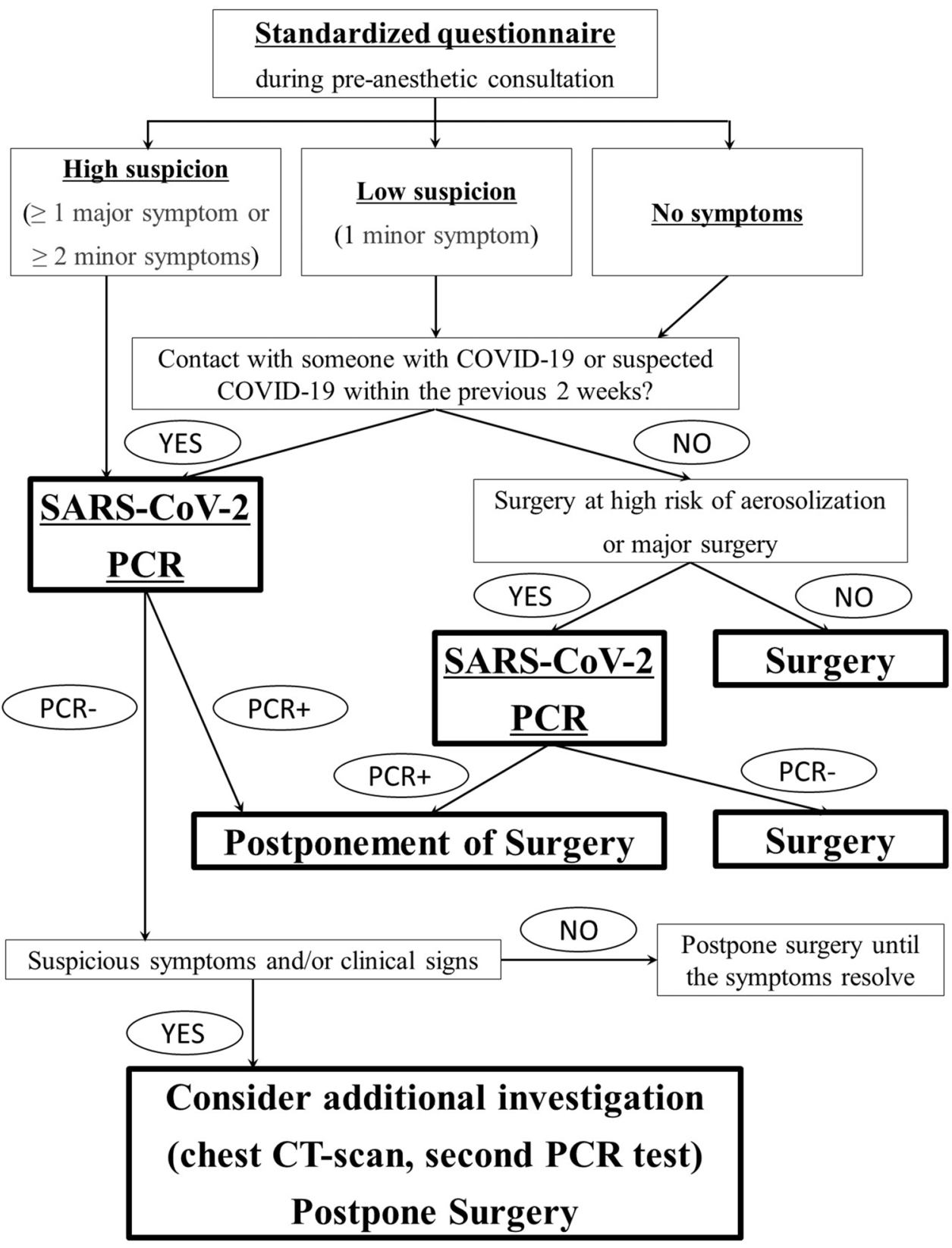

regarding the preoperative assessment of COVID-19 status: one for scheduled and the other for emergency surgery [14]. Figure 1 shows the proposed algorithm for the preoperative assessment of patients undergoing scheduled surgery [14]. This algorithm showed that, in patients with symptoms suggestive of COVID-19 (one or more than one major symptoms, or two or more than two minor symptoms, assessed by the standardized questionnaire), surgery should be postponed until the PCR test results for SARS-CoV-2 are obtained. If the PCR test result is positive, surgery should be postponed until at least 2 weeks after COVID-19 symptom. Wei et al. [17] reported two immunocompromised patients with severe COVID-19 pneumonia. One patient generated specific anti-immunoglobulin $\mathrm{G}$ antibodies of SARS-CoV-2 after 2 weeks of treatment, and could discharge from the hospital on day 42. However, the other failed to obtain specific antibodies of SARS-CoV-2, and eventually died due to respiratory failure [17]. In immunocompromised patients, the surgery may need to be postponed for longer (at least 24 days), because the time to clear SARS-CoV-2 tends to be longer [14, 18]. After the period of postponement, the SARS-CoV-2 PCR test needs to be repeated.

If the PCR test result is negative, physicians need to consider the possibility a false-negative result. If the patient still has suspicious symptoms, additional examinations are necessary. Chest computed tomography (chest-CT) can rule out 
COVID-19 with a high negative predictive value ( 90\%) $[19,20]$. Giannitto et al. [20] performed a prospective study investigating 337 patients with a high likelihood of COVID19 underwent both of PCR test and chest-CT examination. In this population, 87 patients had a negative result on first PCR test, in which 68 patients (78\%) received second repeat PCR tests. Interestingly, the first PCR test provided a falsenegative result in 20 patients (from 68 patients receiving second PCR tests), while only six patients had a false-negative result in chest-CT. For patients who are not able to undergo chest-CT examination, lung ultrasound examination can be an effective measure to detect COVID-19 pneumonia. A previous study [21] reported that lung ultrasound had a good reliability in detecting COVID-19 pneumonia with a sensitivity and a specificity of 68 and 79\%, respectively. The guidelines recommended that a second repeat PCR test should also be considered, which should be performed by trained physicians [14]. Additionally, COVID-19 serology can be useful for patients who have been symptomatic for over 1 week [22]. After infection, most patients generate a serologic response with specific anti-immunoglobulin $\mathrm{G}$ antibodies of SARS-CoV-2 (median time: 11-14 days), which depends on several factors [23]. Patients with mild symptoms and immunocompromised patients may not obtain an adequate antibody response [24]. Positive serology results just indicate that the patient has been infected with SARS-CoV-2, but do not reveal the date of infection or the current infection status. If all these tests are negative, surgery should be postponed until after all symptoms have disappeared.

The guidelines by the French Society of Anaesthesia and Intensive Care Medicine recommended that patients with a low likelihood of SARS-CoV-2 infection (with one minor symptom or no symptoms assessed using the standardized questionnaire) should undergo preoperative SARS-CoV-2 PCR test if they have had close contact with someone having COVID-19 [14]. Additionally, patients undergoing surgical procedures with high risks of aerosolization and those undergoing major surgery should undergo preoperative SARSCoV-2 PCR tests. Surgeries with high risks of aerosolization include otorhinolaryngological surgery, thoracic surgery, and neurosurgery (base of the skull), which require opening of the airways [14]. Major surgeries include cardiovascular surgery, abdominal surgery, and organ transplantation, which have an above average respiratory effect, and can cause the incidence of respiratory complications after surgery. The PCR test needs to be performed the day (at least 2 days) before surgery to minimize the possibility of SARS-CoV-2 transmission after the test.

\section{Preoperative strategy for coronavirus disease in patients undergoing emergency surgery}

Figure 2 shows the proposed algorithm by the French Society of Anaesthesia and Intensive Care Medicine for the preoperative assessment of patients undergoing emergency surgery [14]. This algorithm showed that, among patients undergoing emergency surgery, those with symptoms suggestive of COVID-19 (one or more than one major symptoms, or two or more than two minor symptoms assessed using the standardized questionnaire) should undergo SARS-CoV-2 PCR tests [14]. As with patients undergoing scheduled surgery, those having had close contact with someone with COVID-19 should undergo a PCR test, regardless of the presence of suspicious symptoms. Patients undergoing surgical procedures with high risks of aerosolization and those undergoing major surgery should also undergo preoperative PCR tests. However, the surgery can be performed without waiting for PCR test results [14]. In this situation (especially while awaiting PCR test results), the guidelines recommended that medical staff should wear full personal protective equipment (long-sleeve gown, head cap, gloves, N95 mask, and face shield) to prevent SARS-CoV-2 transmission [14]. All patients should be carefully traced during the postoperative period until their PCR test results are obtained. If possible, regional anesthesia maintaining spontaneous breathing (with a surgical mask) can be considered an alternative technique, as there is an increased risk of transmission during airway management in general anesthesia [5].

\section{Future directions}

Considering that SARS-CoV-2 is transmitted via droplets and secretions, anesthesiologists have high infection risks. The algorithm shown in this review is just one proposal, and local algorithms considering the local COVID-19 epidemic status should take precedence over this algorithm. To date, insufficient information regarding SARS-CoV-2 transmission during the perioperative period is available. Further studies regarding SARS-CoV-2 infection in patients undergoing surgery are needed, and new data from these studies may change the diagnostic algorithm of the preoperative assessment for scheduling surgery. 
Fig. 2 Preoperative assessment of patients undergoing emergency surgery. Adapted from Velly et al. 2020 [13]. Abbreviations: COVID-19 coronavirus disease, $P C R$ polymerase chain reaction, $S A R S$ - $C o V-2$ severe acute respiratory syndrome coronavirus 2

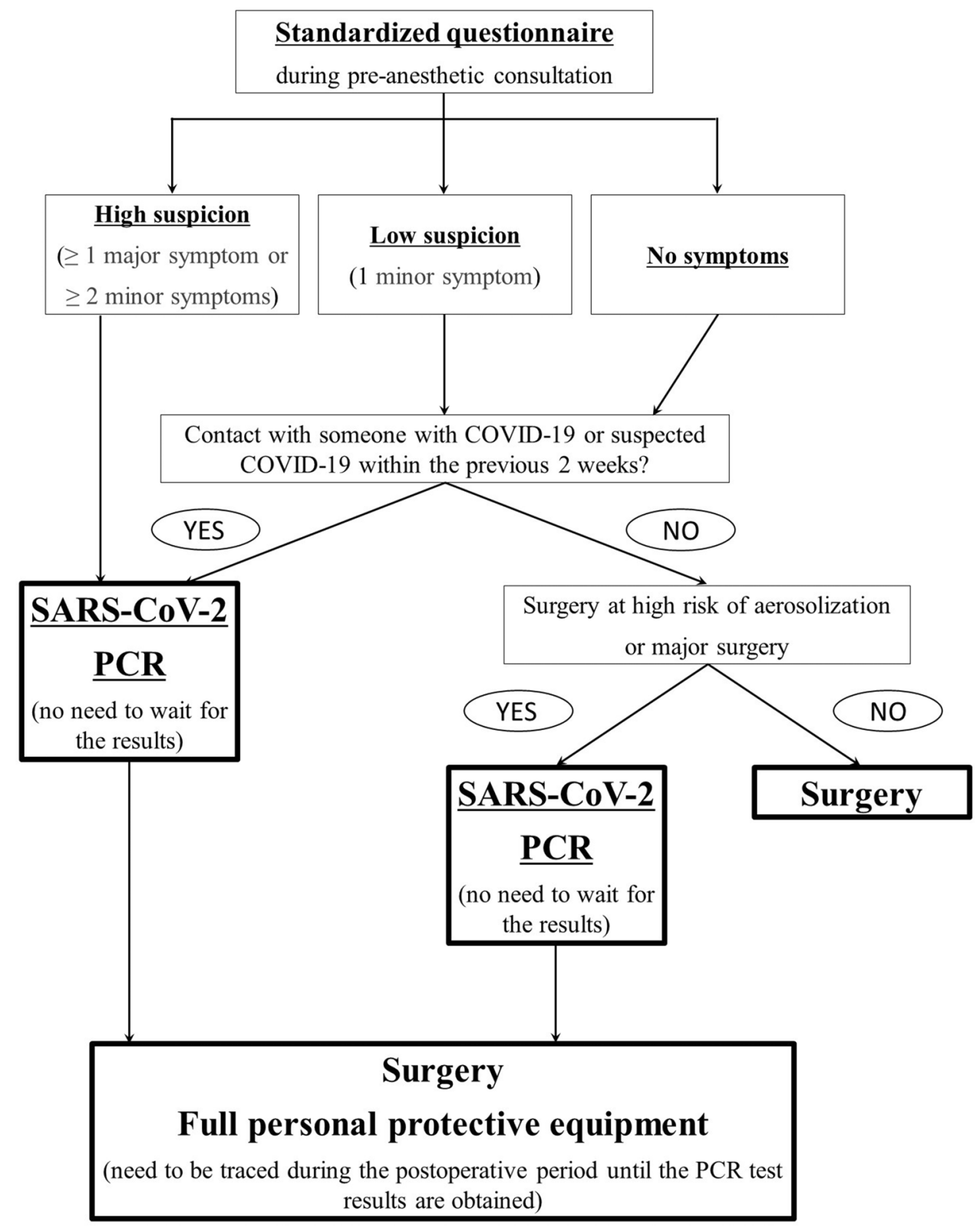

\section{Compliance with ethical standards}

Conflict of interest K.S. has received speaker fees from Edwards Lifesciences and Otsuka Pharmaceutical Factory.

\section{References}

1. Erbabacan E, Özdilek A, Beyoğlu ÇA, Altındaş F. Perioperative anaesthetic management of confirmed or suspected COVID-19 patients. Turk J AnaesthesiolReanim. 2020;48:180-7.
2. Hirata N, Yamakage M. Cardiovascular considerations for anesthesiologists during the COVID-19 pandemic. J Anesth. 2020;3:1-5. https://doi.org/10.1007/s00540-020-02852-1.

3. Chan JF-W, Yuan S, Kok K-H, To KK-W, Chu H, Yang J, Xing F, Liu J, Yip CC-Y, Poon RW-S, Tsoi H-W, Lo SK-F, Chan K-H, Poon VK-M, Chan W-M, Ip JD, Cai J-P, Cheng VC-C, Chen H, Hui CK-M, Yuen K-Y. A familial cluster of pneumonia associated with the 2019 novel coronavirus indicating person-to-person transmission: a study of a family cluster. Lancet. 2020;395:514-23.

4. Hirota K. Air contamination with SARS-CoV-2 in the operating room. J Anesth. 2020;19:1-4. https://doi.org/10.1007/s0054 0-020-02814-7.

5. Hotta K. Regional anesthesia in the time of COVID-19: a minireview. J Anesth. 2020;25:1-4. https://doi.org/10.1007/s00540-02002834-3. 
6. Collaborative C. Mortality and pulmonary complications in patients undergoing surgery with perioperative SARS-CoV-2 infection: an international cohort study. Lancet. 2020;396:27-38.

7. Woloshin S, Patel N, Kesselheim AS. False negative tests for SARS-CoV-2 infection-challenges and implications. N Engl J Med. 2020;383:e38.

8. Arevalo-Rodriguez I, Buitrago-Garcia D, Simancas-Racines D, Zambrano-Achig P, Del Campo R, Ciapponi A, Sued O, Martinez-García L, Rutjes AW, Low N, Bossuyt PM, Perez-Molina JA, Zamora J. False-negative results of initial RT-PCR assays for COVID-19: a systematic review. PLoS ONE. 2020;15:e0242958.

9. Kim H, Hong H, Yoon SH. Diagnostic performance of CT and reverse transcriptase polymerase chain reaction for coronavirus disease 2019: a meta-analysis. Radiology. 2020;296:E145-55.

10. Masui K. Interpretation of laboratory tests for prevention of the SARS-CoV-2 transmission. J Anesth. 2020. https://doi. org/10.1007/s00540-020-02872-x.

11. American Society of Anesthesiologist, Anesthesia Patient Safety Foundation. The ASA and APSF joint statement on perioperative testing for the COVID-19 virus. https://www.asahq.org/about-asa/ newsroom/news-releases/2020/04/asa-and-apsf-joint-statementon-perioperative-testing-for-the-covid-19-virus.

12. Abola RE, Schwartz J, Forrester JD, Gan TJ. A practical guide for anesthesia providers on the management of COVID-19 patients in the Acute Care Hospital. AnesthAnalg. 2020. https://doi. org/10.1213/ANE.0000000000005295.

13. Chen X, Liu Y, Gong Y, Guo X, Zuo M, Li J, Shi W, Li H, $\mathrm{Xu} \mathrm{X}$, Mi W, Huang Y. Perioperative management of patients infected with the novel coronavirus: recommendation from the Joint Task Force of the Chinese Society of Anesthesiology and the Chinese Association of Anesthesiologists. Anesthesiology. 2020;132:1307-16.

14. Velly L, Gayat E, Quintard H, Weiss E, De Jong A, Cuvillon P, Audibert G, Amour J, Beaussier M, Biais M, Bloc S, Bonnet MP, Bouzat P, Brezac G, Dahyot-Fizelier C, Dahmani S, de Queiroz M, Di Maria S, Ecoffey C, Futier E, Geeraerts T, Jaber H, Heyer L, Hoteit R, Joannes-Boyau O, Kern D, Langeron O, Lasocki S, Launey Y, le Sache F, Lukaszewicz AC, Maurice-Szamburski A, Mayeur N, Michel F, Minville V, Mirek S, Montravers P, Morau E, Muller L, Muret J, Nouette-Gaulain K, Orban JC, Orliaguet G, Perrigault PF, Plantet F, Pottecher J, Quesnel C, Reubrecht V, Rozec B, Tavernier B, Veber B, Veyckmans F, Charbonneau H, Constant I, Frasca D, Fischer MO, Huraux C, Blet A, Garnier M. Guidelines: anaesthesia in the context of COVID-19 pandemic. Anaesth Crit Care Pain Med. 2020;39:395-415.

15. Oda Y. COVID-19: review of case reports. J Anesth. 2020;12:1-4. https://doi.org/10.1007/s00540-020-02825-4.
16. Sheleme T, Bekele F, Ayela T. Clinical presentation of patients infected with coronavirus disease 19: a systematic review. Infect Dis (Auckl). 2020;13:1178633720952076.

17. Wei J, Zhao J, Han M, Meng F, Zhou J. SARS-CoV-2 infection in immunocompromised patients: humoral versus cell-mediated immunity. J Immunother Cancer. 2020;8:e000862.

18. Taha M, Sharma A, Taha M, Samavati L. Coronavirus disease 2019 in immunocompromised organ transplant recipients: a case report and review of the literature. Transplant Proc. 2020;52:2698-702.

19. Chetan MR, Tsakok MT, Shaw R, Xie C, Watson RA, Wing L, Peschl H, Benamore R, MacLeod F, Gleeson FV. Chest CT screening for COVID-19 in elective and emergency surgical patients: experience from a UK tertiary centre. ClinRadiol. 2020;75:599-605.

20. Giannitto C, Sposta FM, Repici A, Vatteroni G, Casiraghi E, Casari E, Ferraroli GM, Fugazza A, Sandri MT, Chiti A, Luca B. Chest CT in patients with a moderate or high pretest probability of COVID-19 and negative swab. Radiol Med. 2020;125:1260-70.

21. Fonsi GB, Sapienza P, Brachini G, Andreoli C, De Cicco ML, Cirillo B, Meneghini S, Pugliese F, Crocetti D, Fiori E, Mingoli A. Is lung ultrasound imaging a worthwhile procedure for severe acute respiratory syndrome coronavirus 2 pneumonia detection? J Ultrasound Med. 2020. https://doi.org/10.1002/jum.15487.

22. Azar MM, Shin JJ, Kang I, Landry M. Diagnosis of SARS-CoV-2 infection in the setting of cytokine release syndrome. Expert Rev MolDiagn. 2020;20:1087-97.

23. Long QX, Liu BZ, Deng HJ, Wu GC, Deng K, Chen YK, Liao P, Qiu JF, Lin Y, Cai XF, Wang DQ, Hu Y, Ren JH, Tang N, Xu YY, Yu LH, Mo Z, Gong F, Zhang XL, Tian WG, Hu L, Zhang XX, Xiang JL, Du HX, Liu HW, Lang CH, Luo XH, Wu SB, Cui XP, Zhou Z, Zhu MM, Wang J, Xue CJ, Li XF, Wang L, Li ZJ, Wang K, Niu CC, Yang QJ, Tang XJ, Zhang Y, Liu XM, Li JJ, Zhang DC, Zhang F, Liu P, Yuan J, Li Q, Hu JL, Chen J, Huang AL. Antibody responses to SARS-CoV-2 in patients with COVID-19. Nat Med. 2020;26:845-8.

24. Goetz L, Yang J, Greene W, Zhu Y. A COVID-19 patient with repeatedly undetectable SARS-CoV-2 antibodies. J Appl Lab Med. 2020;5:1401-5.

Publisher's Note Springer Nature remains neutral with regard to jurisdictional claims in published maps and institutional affiliations. 